\title{
Infrared Borescopic Analysis of Ignition and Combustion Variability in a Heavy-Duty Natural-Gas Engine
}

\author{
Michael Gross and Ahmet Mazacioglu University of Michigan
}

Justin Kern Bosch

Volker Sick University of Michigan

Citation: Gross, M., Mazacioglu, A., Kern, J., and Sick, V. "Infrared Borescopic Analysis of Ignition and Combustion Variability in a Heavy-Duty Natural-Gas Engine,” SAE Technical Paper 2018-01-0632, 2018, doi:10.4271/2018-01-0632.

\section{Abstract}

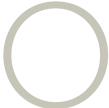

ptical imaging diagnostics of combustion are most often performed in the visible spectral band, in part because camera technology is most mature in this region, but operating in the infrared (IR) provides a number of benefits. These benefits include access to emission lines of relevant chemical species (e.g. water, carbon dioxide, and carbon monoxide) and obviation of image intensifiers (avoiding reduced spatial resolution and increased cost). Highspeed IR in-cylinder imaging and image processing were used to investigate the relationships between infrared images, quantitative image-derived metrics (e.g. location of the flame centroid), and measurements made with in-cylinder pressure transducers (e.g. coefficient of variation of mean effective pressure). A 9.7-liter, inline-six, natural-gas-fueled engine was modified to enable exhaust-gas recirculation (EGR) and provide borescopic optical access to one cylinder for two high-speed infrared cameras. A high-energy inductively coupled ignition system delivered $140 \mathrm{~mJ}$ of energy during each spark event. The engine was operated at $1000 \mathrm{rev} / \mathrm{min}$ and an indicated mean effective pressure of 6.8 bar over a range of air/fuel equivalence ratios, $\lambda$, (1 to 1.6) and EGR rates ( $2 \%$ to $23 \%$ ). Strong emission lines of water are present in the sensitivity band of the cameras $(1.0$ to $1.7 \mu \mathrm{m})$ and can be used as a proxy for the flame front and burned-gas regions. Images were recorded every 5.5 degrees of crank angle (CAD); multiple measurements were interleaved to provide statistical information every 0.5 CAD. The greater cyclic variation resulting from lean/dilute operation is apparent in the images; the image-derived metrics measured early in the cycle correlate strongly with pressure-derived metrics measured later. Centroids calculated from the images show that flames farther from the head and spark plug yield better combustion, which is not evident in the pressure data.

\section{Introduction}

ptical diagnostic techniques; including laserinduced fluorescence, laser-induced incandescence, particle-image-velocimetry, shadowgraphy, schlieren imaging, and others; have been used for decades to study combustion $[1, \underline{2}]$. The vast majority of these measurements have been performed in or near the visible band (usually described as wavelengths from 400 to $700 \mathrm{~nm}$ ), due to the maturity of imaging technology in this band. Silicon is a well developed material system, thanks to its use in computer processors, and it is sensitive to wavelengths from $\sim 400$ to $\sim 1100 \mathrm{~nm}[\underline{3}]$. (An optical filter is used to reject the IR when silicon is used to detect only visible.) Silicon image sensors offer the highest number of pixels, the smallest pixels, the highest frame rates, and the lowest cost available. Lens technology is also well developed for the visible band, since consumer imaging is almost exclusively done in that band. A number of different types of glass have been developed with different refractive indices and dispersions, allowing achromatic (non-dispersive) compound lenses to be assembled.

By contrast, infrared imaging technology is less well developed. Materials for the sensors and lenses become more exotic and expensive as the wavelength increases. However, imaging in the IR offers some crucial benefits that make it worthwhile for some applications, such as combustion research. For example, the near-blackbody radiation produced by soot [4] is much stronger in the IR than the visible for combustion-relevant temperatures. More importantly, the relevant chemical species exhibit many narrow emission lines in the bands covering 1.0 to $5.5 \mu \mathrm{m}[\underline{5}, \underline{6}, \underline{7}]$. Of particular relevance for this document, the strong emission lines of water (broadened into bands by the high in-cylinder temperature and pressure) located between $\sim 1.1$ and $\sim 1.8 \mu \mathrm{m}$ can be used as a proxy for the flame front and burned-gas region. Although water is present in the intake charge due to atmospheric humidity, it is found at higher concentration and higher temperature in the volume where combustion is occurring or 
has occurred. Because the IR emission lines are so strong, when performing passive (non-illuminated) imaging, it is often possible to collect sufficient signal without using an intensifier. Operating without an intensifier is advantageous, since intensifiers are expensive, delicate, and reduce spatial resolution.

Just as there are trade-offs between visible and IR imaging, there are also trade-offs for different types of optical access. Optical engines provide the best available access to the combustion chamber, but optical engines are expensive, limit the peak pressure available, restrict engine speed, can tolerate only short runtimes, and require extensive maintenance. Borescopes provide much more restricted optical access, but they can be installed in an otherwise production engine operating a normal speeds and pressures. The work described here was part of a larger project to investigate ignition in naturalgas-fueled heavy-duty engines under lean/dilute conditions. Because it was impossible to replicate the pressures and dimensions of this engine using the available optical engines $[\underline{8}, \underline{9}]$, it was decided to install borescopes in the metal development engine. Given the limited optical access, the IR band was chosen to maximize the signal.

The purpose of this work was to develop diagnostics based on high-speed IR borescopic imaging of the early flame kernel that not only correlate with pressure-based measurements but also provide information about the eventual heat release of each cycle and of the cycle-to-cycle variation (CCV) earlier in the cycle than pressure transducers can. CCV is important to understand, because it can lead to engine damage and the variations in engine output torque that result from it affect drivability $[\underline{10}, \underline{11}, \underline{12}, \underline{8}]$. In a companion paper, we apply the image-based diagnostics to study the effect of different ignition systems on flame development and CCV in this engine [13].

\section{Experimental Setup and Procedure}

\section{Engine and Instrumentation}

The test engine was a Weichai WP10NG, an inline-six-cylinder engine with a bore $\mathrm{x}$ stroke of $126 \times 130 \mathrm{~mm}$, a displacement of $9,726-\mathrm{cm}^{3}$, two valves per cylinder, and central-point injection of natural gas. This engine had a separate cast-steel head for each cylinder. The turbocharger was replaced with a Bosch Mahle Turbo Systems 110 Series model, and the intake/exhaust plumbing was modified to enable high-pressure exhaust-gas recirculation. An AVL pressure transducer was installed in each cylinder. While the production form of this engine used coils delivering $65 \mathrm{~mJ}$ of energy during each spark, for these experiments, the engine was equipped with $140-\mathrm{mJ}$ coils. The standard spark plugs were retained.

In this work, the EGR rate is defined to be the ratio of background-corrected intake $\mathrm{CO}_{2}$ concentration to background-corrected exhaust $\mathrm{CO}_{2}$ concentration. The measured EGR rate with the EGR valve completely closed was in the range of $2-4 \%$. This nonzero value resulted from residual $\mathrm{CO}_{2}$ in the intake manifold from leakage past the EGR valve, and possibly re-aspirated exhaust gases in the intake manifold. No operating conditions with truly 0\% EGR were recorded.

\section{Optical Access and Imaging Equipment}

The rearmost cylinder (Cylinder 6), being the most accessible, was modified to provide optical access for two cameras; two borescope sleeves were installed in the head, and reliefs were cut into the top of the piston to provide clearance for the tips of the borescopes. Figure 1 is a drawing of the engine, with cameras and associated hardware, viewed from two directions. Due to the limited options resulting from the positions of the valves, spark plug, ports, coolant passages, and intake manifold, the borescopes could not be positioned $90^{\circ}$ apart; the angle between the borescopes, measured about the vertical axis, was $\sim 70^{\circ}$. The relative positions and orientations of the borescopes and spark plug are clearly visible in Figure 2. Initial imaging showed reflections of the flame kernel from the piston. To reduce these reflections, the piston top and head were painted with flat black VHT Flameproof hightemperature coating.

The borescopes, sleeves, and related hardware were purchased from AVL. The borescopes measure $7 \mathrm{~mm}$ in outside diameter, provide an angular field of view of $70^{\circ}$, and are optimized to focus light between 1.0 and $1.7 \mu \mathrm{m}$. The borescopes were cooled with air at $\sim 6$ bar pressure that was first passed through an AVL filter unit, which removed particles that could clog the cooling channels. The borescopes were protected from the heat and pressure in the chamber by

FIGURE 1 Drawings of the engine and optical equipment, viewed from two directions, showing the positions of the cameras and borescopes.

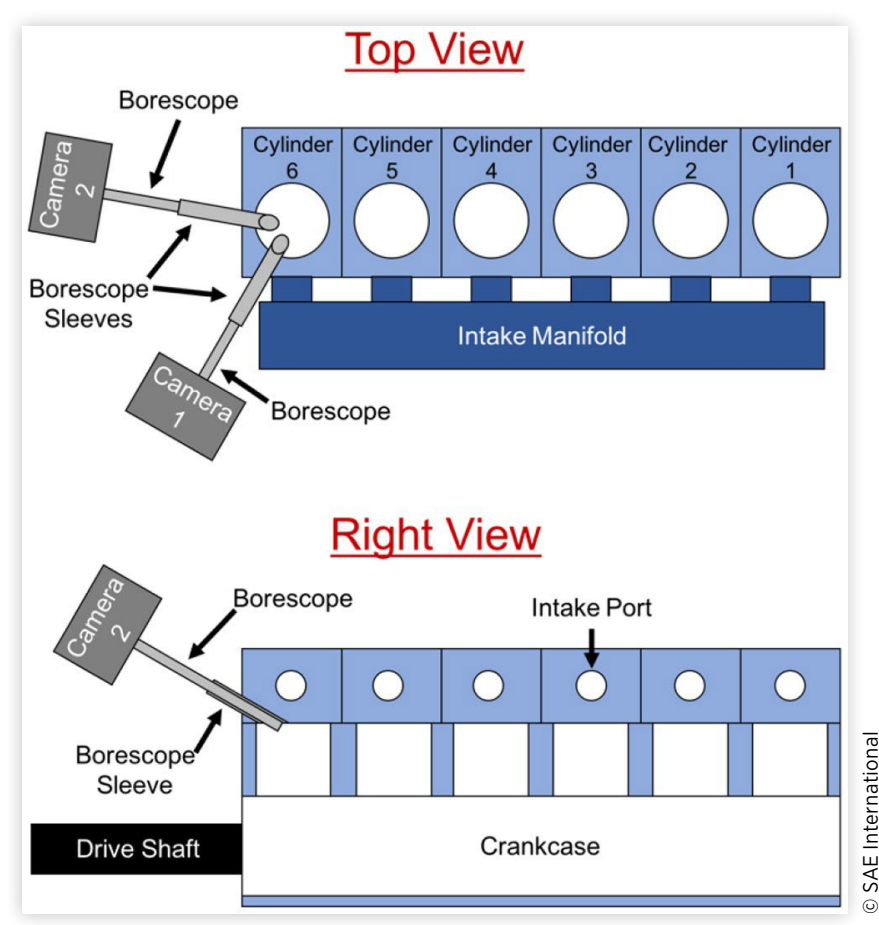

(c) 2018 SAE International. All Rights Reserved. 
FIGURE 2 A photograph of the modified head with borescopes installed. Note the two views provided by Cameras 1 and 2.

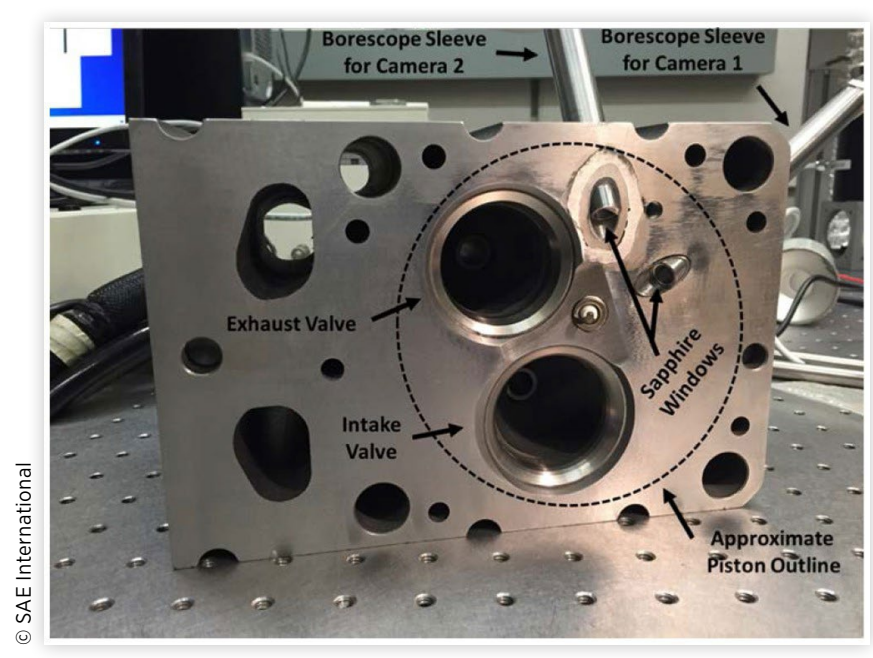

sapphire windows. The cameras were rigidly held in place relative to the engine and borescopes using a combination of AVL-supplied brackets and custom-fabricated parts.

Each camera was a Xenics Cheetah-640-CL with the VisNIR option. The sensor chip has 640 x 512 monochrome pixels with 12 bits of depth, and it is sensitive to wavelengths between $400 \mathrm{~nm}$ and $1.7 \mu \mathrm{m}$ (though maximum sensitivity occurs between 1.0 and $1.6 \mu \mathrm{m}$ ). While the borescopes do transmit visible light (the shorter end of the camera's sensitivity range), the focus is poor in the visible. A 1000-nm longpass filter, placed between each camera and its borescope, was found to provide the best compromise between image brightness and clarity. The camera can operate at frame rates up to $865 \mathrm{~Hz}$ in full-frame mode, but when only a subset of the sensor is used, much higher frame rates are possible. Preliminary imaging indicated that the field of view (FoV) provided by each borescope only occupied $\sim 60 \%$ of the camera sensor in each dimension, so both cameras were windowed to $384 \times 336$ pixels in order to increase the maximum frame rate beyond $1 \mathrm{kHz}$ without sacrificing any useful information.

One-per-cycle and 1440-per-cycle signals were provided to a LaVision PTU-X programmable timing unit, which in turn triggered the cameras to capture images. One instance of LaVision Davis 8 was used to program the timing unit and record images from Camera 1; a second instance of $\mathrm{DaV}$ is captured images from Camera 2.

\section{Engine Calibration}

It was necessary to determine the maximum air/fuel ratio and EGR dilution for each operating point, as lean combustion is desired for improving fuel efficiency, and EGR dilution is desired for improving both efficiency and $\mathrm{NO}_{x}$ emissions. This dilution amount was determined by setting an upper limit on the $\mathrm{CoV}$ (IMEP) for each individual cylinder of between 6-8\%. It should be noted that, despite the modifications for optical access, Cylinder 6 did not suffer the most CCV. The worst CoV(IMEP) values were those of Cylinders 3 and 4 , the middle two cylinders. It is speculated that imperfect air/fuel mixing in the intake manifold may have resulted in less well-mixed or globally leaner mixtures reaching these two cylinders, leading to less consistent cycle-to-cycle combustion. The IMEP and $\mathrm{CoV}(\mathrm{IMEP})$ values reported here are for Cylinder 6 specifically.

For each operating point defined by engine speed, load (IMEP), air/fuel ratio, and EGR rate, the spark timing was adjusted to position the crank angle at which $50 \%$ of the fuel (by mass fraction) has been burned, $\mathrm{MFB}_{50}$, at approximately 9 crank angle degrees after top dead center ( ${ }^{\circ}$ aTDC) for the entire engine. (Throughout this document, $\mathrm{MFB}_{x}$ refers to the crank angle at which $x \%$ mass fraction of the fuel has been consumed.) This target value was determined from engine mapping with ignition timing sweeps at various operating points as the $\mathrm{MFB}_{50}$ that minimized the brake specific fuel consumption.

\section{Data and Image Acquisition}

The operating points discussed here are shown in Table 1. Some results are also included in the table; they are discussed later in this document. All four points were measured at 1000 rev/min with a 6.8-bar IMEP in Cylinder 6. The EGR values of $2-3 \%$ were measured with the EGR valve closed and represent the minimum attainable. Each column of the table is color coded from dark red (the lowest value to dark blue (the highest) in order to make it easier for the reader to compare the operating points.

At each operating point, the in-cylinder pressure and images were recoded over a 100 -cycle period; the statistical metrics reported here were computed over this period. Images were recorded at intervals of 5.5 CAD, corresponding a frame rate of $1.09 \mathrm{kHz}$. In order to provide more finely time-resolved information, ten measurements were made for each operating condition, each with the timing of the first image offset by a

TABLE 1 Engine operating conditions and parameters measured. Engine speed is $1000 \mathrm{rev} / \mathrm{min}$; IMEP is 6.8 bar. Cases with 2-3\% EGR occurred with the EGR valve fully closed. Each column is color coded from lowest value (red) to highest (blue). Symbols are defined in the table at the end of the document.

\begin{tabular}{|c|c|c|c|c|c|c|c|c|c|c|c|}
\hline $\begin{array}{l}\text { Air/Fuel } \\
\text { Equiv. } \\
\text { Ratio }\end{array}$ & EGR [\%] & $\begin{array}{l}\text { Spark } \\
\text { Timing } \\
\text { [ªTDC] }\end{array}$ & $\begin{array}{l}\text { CoV(IMEP) } \\
{[\%]}\end{array}$ & $\begin{array}{l}R\left(\mathrm{MFB}_{10},\right. \\
\left.r_{\mathrm{gyr}}\right)\end{array}$ & $\begin{array}{l}R\left(\mathrm{MFB}_{90},\right. \\
\left.r_{\mathrm{gyr}}\right)\end{array}$ & $\begin{array}{l}R\left(M_{F} B_{90}\right. \\
-M B_{10} \\
\left.r_{\text {gyr }}\right)\end{array}$ & $\begin{array}{l}R\left(P_{\max }\right. \\
\left.r_{\text {gyr }}\right)\end{array}$ & $\begin{array}{l}R\left(M_{F} B_{10},\right. \\
S)\end{array}$ & $\begin{array}{l}R\left(M B_{90},\right. \\
S)\end{array}$ & $\begin{array}{l}R\left(\mathrm{MFB}_{90}\right. \\
-\mathrm{MFB}_{10} \\
S)\end{array}$ & $R\left(P_{\max }, S\right)$ \\
\hline 1.0 & 3 & -19.50 & 0.32 & -0.86 & -0.73 & -0.24 & 0.81 & -0.77 & -0.61 & -0.13 & 0.68 \\
\hline \multirow[t]{2}{*}{1.6} & 2 & -35.25 & 0.88 & -0.80 & -0.67 & -0.27 & 0.81 & -0.79 & -0.63 & -0.23 & 0.74 \\
\hline & 10 & -55.50 & 1.6 & -0.76 & -0.47 & 0.03 & 0.73 & -0.84 & -0.52 & 0.03 & 0.77 \\
\hline
\end{tabular}


FIGURE 3 An illustration of the crank angles at which images were recorded. Images from each subset were recorded in a single measurement. Successive subsets were recorded in successive measurements moments later.

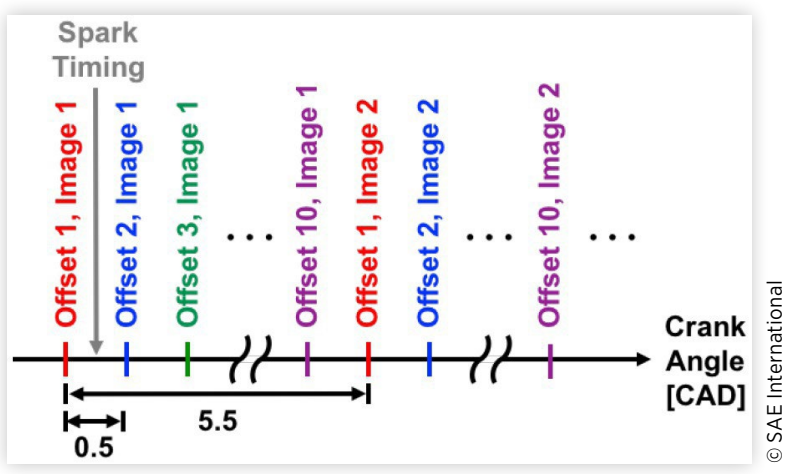

multiple of $0.5 \mathrm{CAD}$, as illustrated in Figure 3. Due to CCV, the images cannot simply be interleaved to fill in the gaps in the imaging. However, since 100 cycles of images were recorded at each offset, they can be used to provide statistical information at 0.5-CAD intervals, as will be described in the next section. During each cycle, images were recorded from just before spark timing to the point at which the FoV was nearly filled. Operating points that were lean or diluted necessitated earlier ignition timing and burned more slowly, thus requiring longer imaging periods during each cycle.

\section{Image Processing and Analysis}

The view of the top of the flame was more obstructed by the head in Camera 2's images, making computing metrics from these images more challenging. These computations are still being developed, so only images and metrics from Camera 1 are reported here.

The image-processing procedure, illustrated in Figure 4, was carried out with a combination of LaVision $\mathrm{DaV}$ is and MathWorks MATLAB. Although the cameras have monochrome sensors, false color was applied in many images presented here to aid the reader's eye. The first image in Figure $\underline{4}$ is the raw image recorded by Camera 1 at $1000 \mathrm{r} / \mathrm{min}, 6.8 \mathrm{bar}$ IMEP, $\lambda=1.0$, and 3\% EGR. A background image (not shown), recorded immediately before ignition (as indicated in Figure 3), was subtracted, reducing noise and yielding the second image. A median filter with a 3x3-pixel window was applied to smooth the image without compromising the edges as much as a Gaussian filter, yielding the third image. Even with the piston painted matte black, reflections from the piston were still visible in some cases. To reduce these reflections, Otsu's method of thresholding [14] was applied. Values below the threshold were set to 0 ; those above were set to 1 , resulting in the fourth image. The median-filtered image was multiplied by the binarized image to create the thresholded final image.

The collection of all the images recorded at a given crank angle during each 100-cycle measurement is called a "subset" in this document. Each subset of thresholded images was
FIGURE 4 Images from Camera 1 illustrating the image-processing procedure.

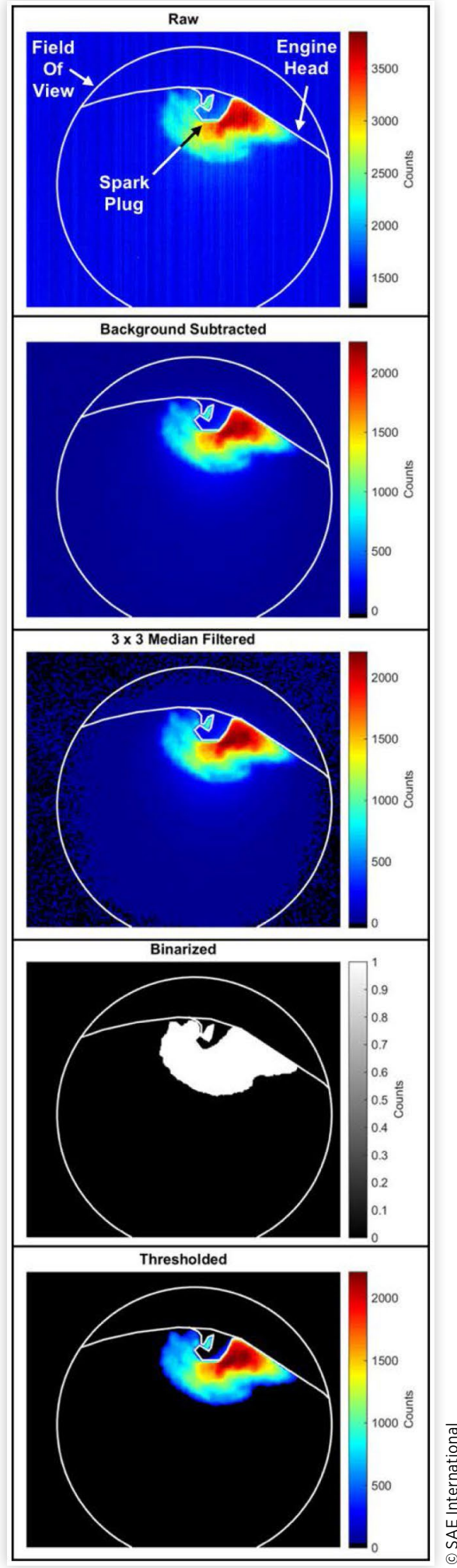

averaged, creating a mean image for each crank angle. The standard deviation of all the binarized images in select subsets was computed. The binarized image was chosen as the source of this calculation in order to avoid complications due to saturation. Using the binarized images also yielded 
standard-deviation images that were automatically normalized to a fixed scale: 0-0.5. A number of quantitative metrics were computed from the single and mean images, as described below.

The total integrated image signal, $S$, was defined as the sum of the counts in all pixels of a thresholded image. $S$, being essentially the total brightness of the flame in the 1.0- to 1.7$\mu \mathrm{m}$ band, should be strongly dependent on the temperature and concentration of water in the flame. It is therefore expected that $S$ should be correlated with the timing of major combustion landmarks.

The location of the flame centroid (center of mass) of each single image and each mean image was computed by weighting each pixel according to its brightness. The centroid is one way to quantify a flame's location. A "centroid cloud" was created for select subsets by marking the centroid of each image with a circle color-coded by the peak pressure, $P_{\max }$, of that cycle. A centroid cloud illustrates the distribution of flame locations for each operating condition and shows where the flames producing the lowest and highest peak pressure were located. Centroid displacement was defined as the distance (in pixels) of the centroid of a given image from the centroid of the mean image. The effective radius (in pixels) of each flame was calculated as the radius of a circle having the same area as the flame. The normalized centroid displacement, $\delta$, was calculated by dividing the centroid displacement of an image by the effective radius of the flame in that image. $\delta$ quantifies how far away a given cycle's flame is from the flame's average position for that operating condition.

$$
I=\sum_{n=1}^{N} \sum_{m=1}^{M} c_{m, n} \times\left[\left(m-y_{c}\right)^{2}+\left(n-x_{c}\right)^{2}\right]
$$

The area moment of inertia (also called the second moment of area), $I$, was computed by the formula shown above for an $M x N$-pixel image, where $c_{m, n}$ is the pixel intensity at $m^{\text {th }}$ row and $n^{\text {th }}$ column and $\left(x_{c}, y_{c}\right)$ is the centroid location. I is a measure of how much "mass" (number of pixels weighted by signal strength) a flame image has and how far that mass is from the centroid.

$$
r_{g y r}=\sqrt{I / A}
$$

Because very different shapes can have the same area and the same moment of inertia, the radius of gyration, $r_{\text {gyr }}$, was computed by the above formula from the area moment of inertia and the total projected area of the flame, $A$, in order to quantify the differences in flame shape. The radius of gyration is the radius of a point mass with the same $I$ as the flame in the image; it is equal to the root-mean-square distance of each pixel (weighted by its brightness) from the centroid. For objects in general, $r_{\text {gyr }}$ is a measure of how compact or extended the object is. A circle will have a small radius of gyration, while a rectangle of the same area with an aspect ratio of, e.g., 10:1 will have a larger $r_{\text {gyr }}$. A flame whose image has a larger radius of gyration would be expected to have a higher ratio of area to volume, in other words to be more stretched or wrinkled, and to burn more quickly.

Each borescope contains a fish-eye lens at its tip, which provides the widest possible FoV but also distorts the images.
As a result, calibrating the spatial dimensions, so that distances (e.g., $\delta$ ) can be reported in $\mathrm{mm}$ rather than in pixels, is not as straightforward as applying a linear pixel-to-mm scaling. Instead, three-dimensional dewarping must be performed. That processing has not yet been undertaken, and all distances were calculated in pixels. It is expected that proper dewarping, once performed, will only strengthen the relationship between the image-derived and pressure-derived metrics described in the Results sections, so the conclusions drawn in that section remain valid even without calibration.

In order to investigate whether the IR images of the early flame kernel agreed with and predicted measurements based on the in-cylinder pressure, the Pearson correlation coefficient, $R$, was calculated for several combinations of an imagebased metric (e.g. $r_{\text {gyr }}, \delta$ ) computed at an early crank angle and a later metric (e.g., $\mathrm{MFB}_{10}$ or $P_{\max }$ ) computed from in-cylinder pressure measurements in Cylinder 6 (and thus specific to that cylinder). It was not reasonable to compare the image-based metrics for different operating points at the same crank angle, since the flame development at any one crank angle would be very dissimilar between the points. Likewise, using a fixed crank-angle delay relative to the spark timing would not work for all conditions. For this document, the mean $\mathrm{MFB}_{00.5}$ in Cylinder 6 for a given operating condition, labeled mean $\left(\mathrm{MFB}_{00.5}\right)$ here, was chosen as the time at which the image-based measurements would be computed for the Pearson calculation. This timing provided a more consistent level of flame development and ensured sufficient delay between the image- and pressure-based measurements for the results to be of interest. The symbol $R(x, y)$ is used in this document to represent the correlation between image metric $x$, measured at mean $\left(\mathrm{MFB}_{00.5}\right)$, and pressure metric $y$.

In order to compare images at multiple crank angles for different operating conditions, it was necessary to determine "normalized crank angles" at which flame development was comparable across conditions. Six crank angles evenly spaced between spark timing and mean $\left(\mathrm{MFB}_{00.5}\right)$ were computed for each condition, and these crank angles were labeled $\theta_{x / 5}$ where $x$ indicated the fraction of angle between spark and mean $\left(\mathrm{MFB}_{00.5}\right)$, as shown in Figure 5 .

\section{Results and Discussion}

Figure 6 presents the mean images (computed over 100 cycles) at five normalized crank angles $\left(\theta_{1 / 5}\right.$ through $\left.\theta_{5 / 5}\right)$ for all four operating conditions. The conditions are shown in the order

FIGURE 5 The normalized crank angles, $\theta_{x / 5}$, at which images from different conditions were compared.

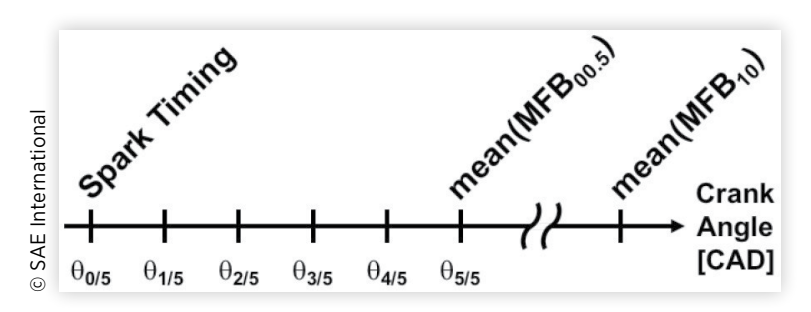


FIGURE 6 Mean images from Camera 1 for five crank angles for four operating conditions, all at an engine speed of 1000 rev/ min and an IMEP of 6.8 bar.

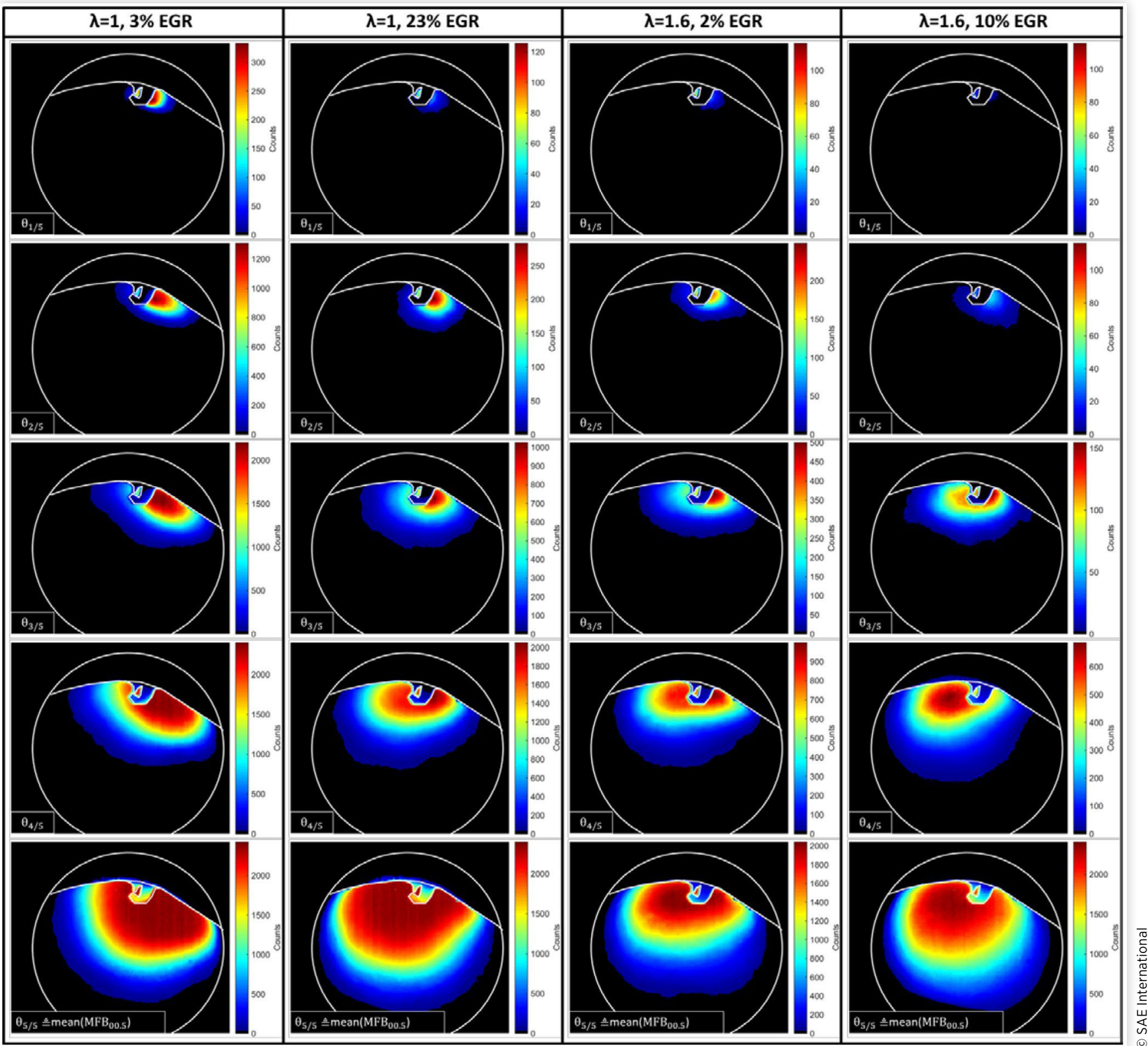

of increasing IMEP variability-see Table 1-from left to right. As expected, mean images of all four cases at $\theta_{5 / 5}$ look similar in terms of flame size and intensity distribution, because they all correspond to the same combustion phase $\left(\mathrm{MFB}_{00.5}\right)$. However, the discrepancy in flame-kernel size between operating conditions becomes increasingly apparent for increasingly early normalized crank angles. Especially at $\theta_{1 / 5}$, the kernel size is clearly largest for the stoichiometric and minimally diluted case, whereas it is the smallest for the lean and diluted case. This result indicates that the initial laminar flame-kernel growth regime is extended, and thus flame wrinkling is delayed, with excess air and dilution. Another observation from Figure 6 is that the flame location and orientation differ substantially from case to case across any given $\theta_{x / 5}$. The primary reason is the discrepancy in spark timing (Table 1).
Since the optimized spark timing is different for each case, the flow (tumble and swirl) and pressure conditions during spark and early kernel growth are also different, which result in differences in flame-growth patterns.

The standard deviation of the binarized images computed at the same normalized crank angles are presented in Figure 7. These images visualize the variation in location of the flame boundaries over 100 cycles. For all four cases, the curved red band indicating flame-boundary variability widens as the combustion phase progresses from $\theta_{1 / 5}$ to $\theta_{5 / 5}$. Comparison of different operating points across the same $\theta_{x / 5}$ reveals that EGR dilution and lean operation, either individually or combined, widen the band of flame-boundary variability. This observed image variability is consistent with the CoV(IMEP) results tabulated in Table 1 , as the cases with higher 
FIGURE 7 Standard deviation of the binarized images from Camera 1 for five crank angles for four operating conditions, all at an engine speed of $1000 \mathrm{rev} / \mathrm{min}$ and an IMEP of $6.8 \mathrm{bar}$.

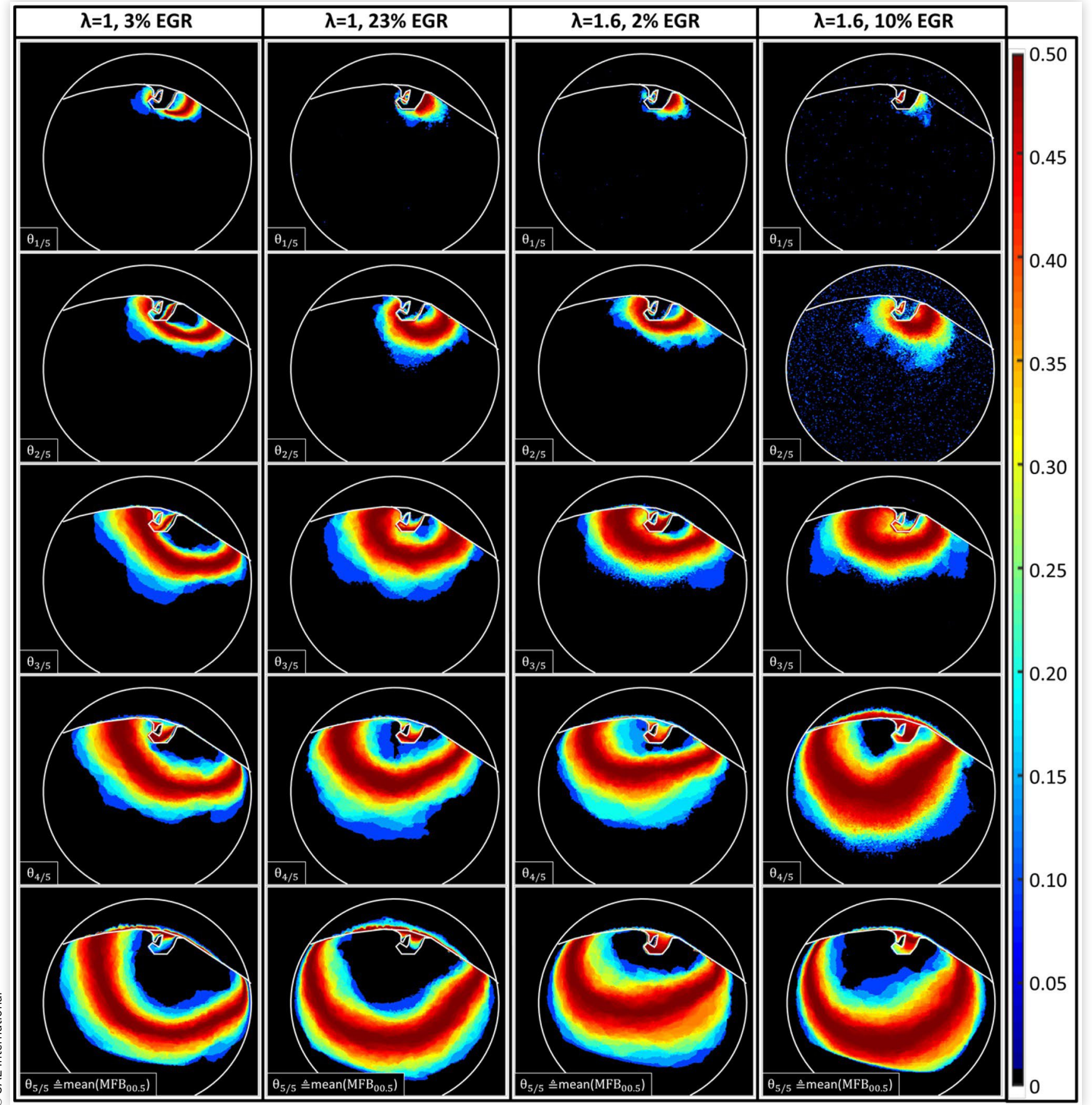

CoV(IMEP) exhibit higher flame boundary variability, and vice versa.

Centroid clouds computed at the same crank angles for all four conditions are shown in Figure 8. Comparing the first column to the second and the third column to the fourth shows that, until about $\theta_{3 / 5}$, the cases with the minimum achievable EGR have smaller clouds than those with $10-23 \%$ EGR. Similarly, comparing the first column to the third shows that, until $\theta_{3 / 5}$, the stoichiometric case produces a smaller cloud than the lean case. This result indicates that both higher EGR rates and leaner air/fuel ratios are associated with less consistent flame location, which agrees with the well documented observation that increasing the EGR rate or $\lambda$ increases CCV. It has been established $[\underline{15}, \underline{16}]$ that the combustion physics occurring between spark timing and $\sim \mathrm{MFB}_{01}$ is the principal source of CCV. Schiffmann et al. [] studied the relationship between the early kernel growth and CCV using a number of optical diagnostics applied to an optically accessible engine. This paper attempts to investigate the same period in a modified production engine via the more limited optical access provided by borescopes and using IR imaging to maximize signal and locate the flame. One approach to 
reliably ignite leaner and more EGR-dilute mixtures is to employ an advanced ignition system, which is the goal of the project of which this work is part. The first results of the impact of igniter choice on the combustion of lean/dilute natural-gas mixtures resulting from the project are described elsewhere [13].

Examining Figure 8 again reveals another pattern, which appears by mean $\left(\mathrm{MFB}_{00.5}\right)$, the bottom row of the figure, for each operating point. For each condition, centroids positioned lower in the plot (farther from the head) very early in the cycle lead to higher peak pressures. This result makes intuitive sense; flames centered farther from the head have more room to grow without being quenched, leading to better combustion. This relationship, though well known, is not provided by the pressure-derived metrics.

Figure 9 is a set of correlation plots, each showing the relationship between four $\mathrm{MFB}_{x}$ values $\left(\mathrm{MFB}_{05}, \mathrm{MFB}_{10}, \mathrm{MFB}_{50}\right.$, and $\mathrm{MFB}_{90}$ ) and the radius of gyration for 100 cycles for all four operating points. Recall that $r_{\mathrm{gyr}}$ is a measure of the degree to which the flame is stretched or elongated. The plots and the Pearson correlation coefficients shown in this figure and tabulated in.

Table 1 indicate that the very early-cycle measurement of the radius of gyration is a good predictor for the timing of

FIGURE 8 Centroid clouds for five crank angles for four operating conditions, all at an engine speed of $1000 \mathrm{rev} / \mathrm{min}$ and an IMEP of 6.8 bar.

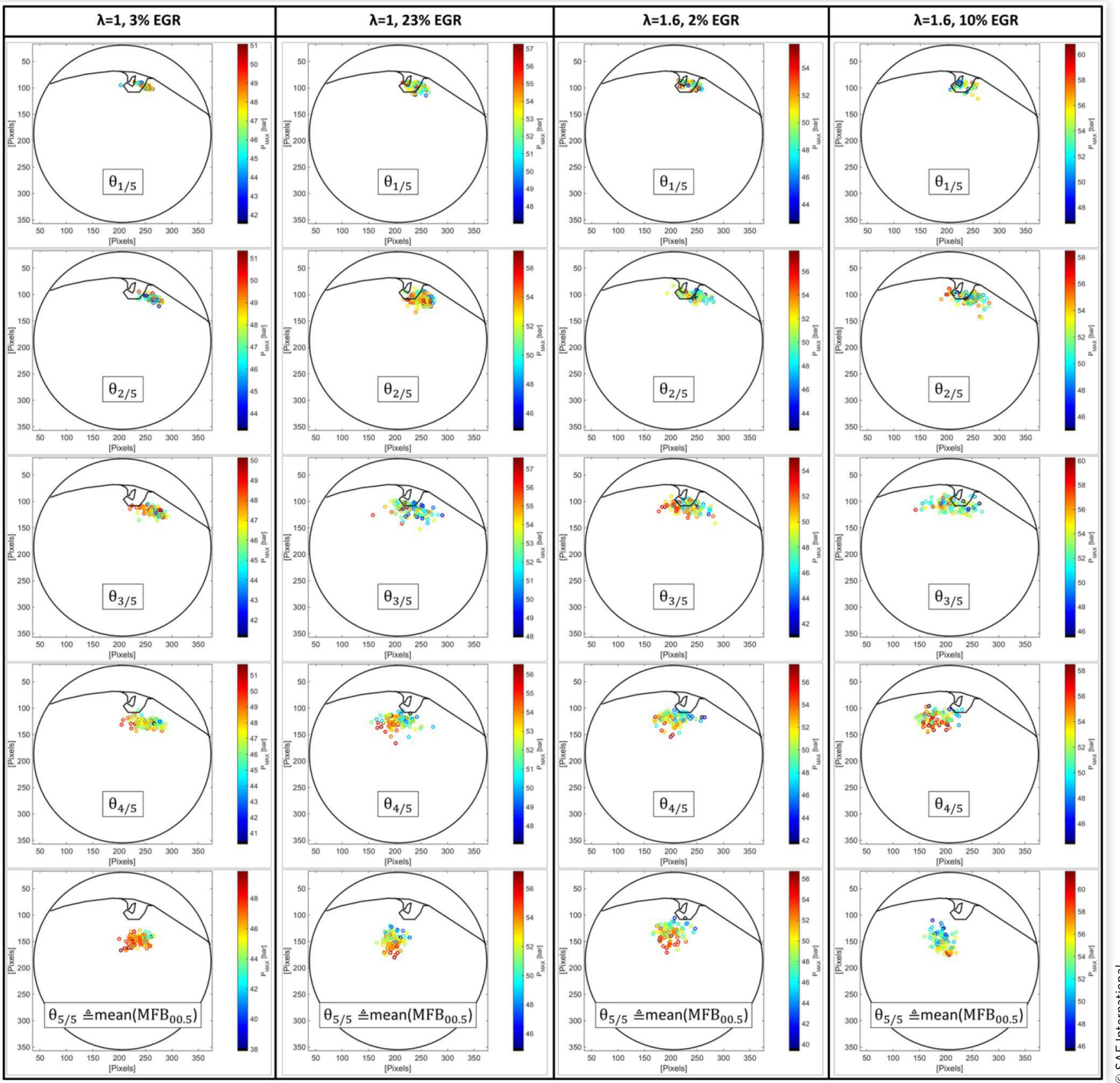


FIGURE $9 \mathrm{MFB}_{05}, \mathrm{MFB}_{10}, \mathrm{MFB}_{50}$, and $\mathrm{MFB}_{90}$ correlated with $r_{\text {gyr }}$ measured at mean(MFB ${ }_{00.5}$ ) for 100 cycles of operation at all four operating conditions.
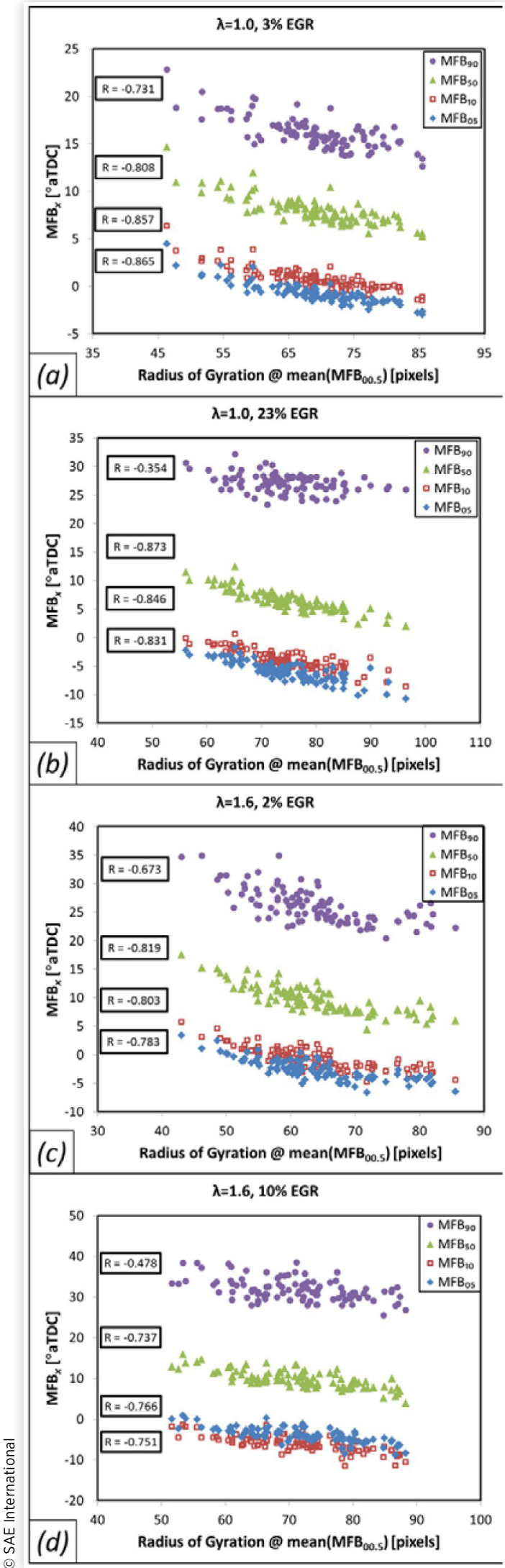

major combustion landmarks up to $\mathrm{MFB}_{50}$, but that this predictive capability is weaker for $\mathrm{MFB}_{90}$ and therefore for combustion duration (defined here to be $\mathrm{MFB}_{90}-\mathrm{MFB}_{10}$ ). Note that the correlation coefficients are negative, meaning a flame whose two-dimensional projection exhibits a smaller $r_{\text {gyr }}$ is correlated with later values for $\mathrm{MFB}_{05}, \mathrm{MFB}_{10}, \mathrm{MFB}_{50}$, and $\mathrm{MFB}_{90}$. This result is consistent with expectations; a flame whose image has a smaller radius of gyration would be expected to be less stretched and wrinkled and therefore to burn more slowly.

Similarly, the rightmost four columns of Table 1, though not plotted, show that the total integrated signal predicts the early flame development through $\mathrm{MFB}_{50}$ well (though not as well as $r_{\text {gyr }}$ does), but likewise does not correlate as strongly with $\mathrm{MFB}_{90}$ and combustion duration. This result is consistent with the interpretation of the integrated signal as closely related to the reaction rate.

Returning to the discussion of radius of gyration, Figure 10 is a correlation plot showing the relationship between the peak in-cylinder pressure and the $r_{\text {gyr }}$ measured at mean $\left(\mathrm{MFB}_{00.5}\right)$ and for all 100 cycles at the four operating points. The $R$ values presented in the legend show that $r_{\text {gyr }}$ measured very early in the cycle can predict the eventual $P_{\max }$, which typically occurs somewhere near $\mathrm{MFB}_{50}$, for all four conditions. In other words, more extended flames burn more quickly and reach higher pressures, which is consistent with expectations.

Figure 11 is a set of correlation plots showing the relationship between normalized centroid displacement and $\mathrm{MFB}_{10}$ for all 100 cycles of the same four operating conditions. Recall that $\delta$ is a measure of how far the position of the flame at one early crank angle in any given cycle differs from the average centroid position over 100 cycles. The dashed line segments are added to aid the eye. For $\delta$ values below $\sim 0.2-0.4$, where the density of data points is relatively high, a pattern is evident in the point distribution; the cycles

FIGURE 10 Peak pressure correlated with radius of gyration measured at mean $\left(\mathrm{MFB}_{00.5}\right)$ for 100 cycles of operation at four operating conditions.

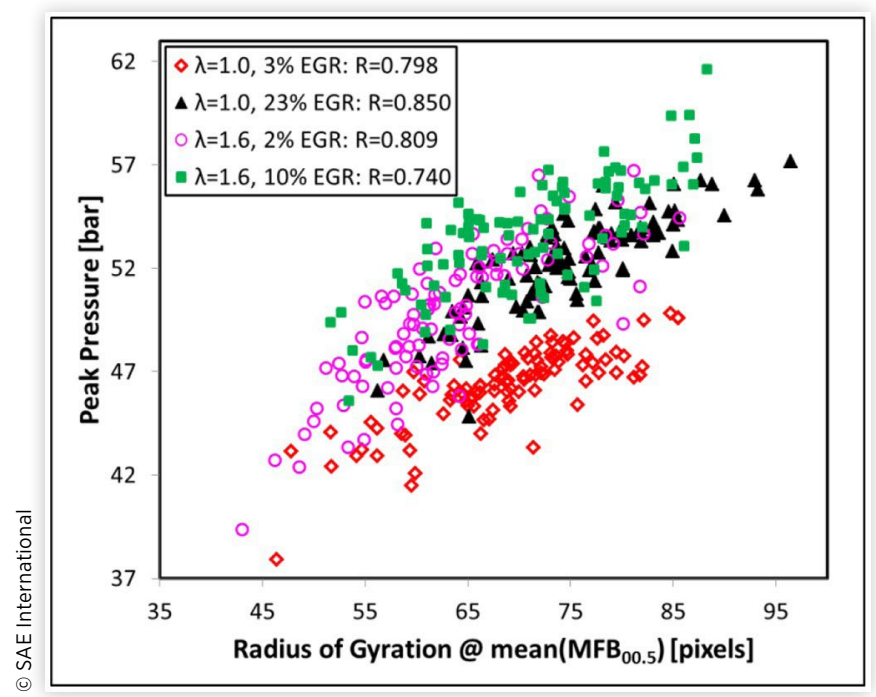


FIGURE $11 \quad M_{10}$ correlated with $\delta$ measured at mean(MFB $\left.{ }_{00.5}\right)$ for 100 cycles at all four operating conditions.

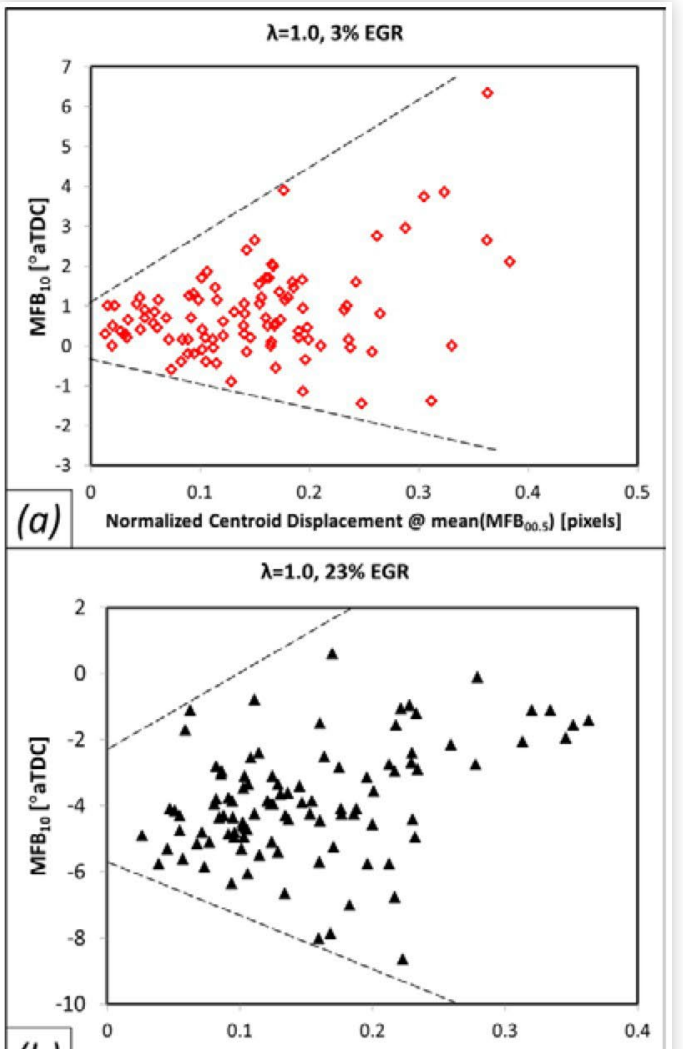

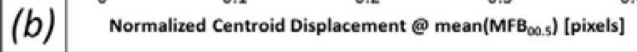
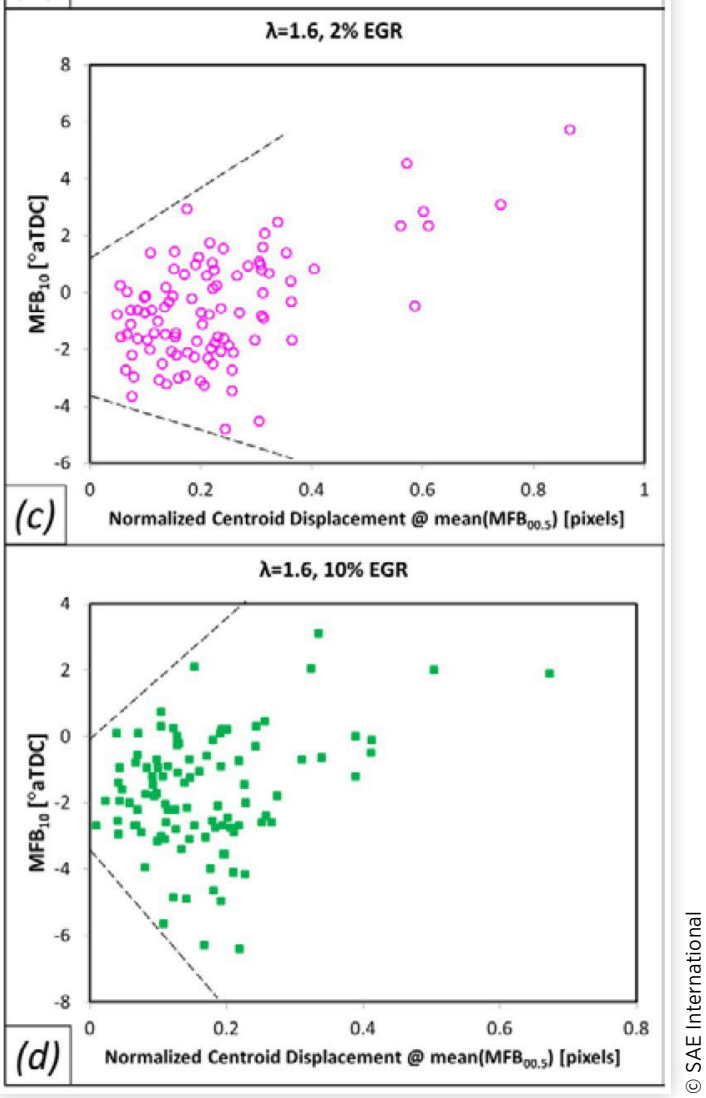

with smaller displacements are more consistent in the time at which they reach $10 \%$ fuel burn. For $\delta$ values greater than $\sim 0.2-0.4$, the density of points is too low for any pattern to be apparent, so no conclusion can be drawn from these few highly displaced cycles. This result indicates that flames that are located farther from the average position result in more inconsistent combustion timing.

\section{Summary and Conclusions}

In-cylinder infrared borescopic imaging of early flame-kernel growth at $1.09 \mathrm{kHz}$ has been described, and the procedure for processing these images and deriving quantitative metrics from them has been explained. These optical techniques were applied, along with standard measurements based on in-cylinder pressure, to a natural-gas-fueled heavy-duty engine burning four different air/fuel mixtures at an engine speed of $1000 \mathrm{rev} / \mathrm{min}$ and an IMEP of $6.8 \mathrm{bar}$.

Imaging in the near infrared and short-wavelength infrared offers several advantages over visible-band optical diagnostics. Since the $\mathrm{H}_{2} \mathrm{O}$ emissions are strong in this spectral region, the need for intensifiers or other additional equipment is eliminated. Additionally, the strength of this emission makes it possible to obtain high-quality flame images through borescopes installed in a production engine, rather than an optically accessible engine, providing access to realistic operating conditions not available in optical engines.

Several relationships between image-based metrics measured very early in the cycle, at mean $\left(\mathrm{MFB}_{00.5}\right)$, and pressure-derived metrics measured much later in the cycle were observed:

- Flames centered further from the head at mean $\left(\mathrm{MFB}_{00.5}\right)$ lead to higher peak pressures, showing that borescopic infrared high-speed imaging can provide guidance for future engine design.

- Flames displaced farther from the mean location at the beginning of combustion exhibited less consistent early flame growth (quantified by $\mathrm{MFB}_{10}$ ) than flames not displaced as far.

- Flames with larger radii of gyration at mean $\left(\mathrm{MFB}_{00.5}\right)$, in other words, flames with more extended shapes (due to high levels of flame stretching or wrinkling), were strongly correlated with higher peak combustion pressure, earlier values of $\mathrm{MFB}_{05}, \mathrm{MFB}_{10}$, and $\mathrm{MFB}_{50}$, and were also correlated (though more weakly) with earlier values of $\mathrm{MFB}_{90}$.

- EGR dilution and lean operation caused an increase in flame-boundary variability in the early flame kernel that is consistent with the IMEP variability.

These results are consistent with expectations, but it is important to note that the image-derived metrics reveal trends earlier in the cycle than they can be detected from the pressure measurements. These metrics therefore have some predictive 
capability that will be further investigated in the future. In conclusion, the measurement technique and image-processing procedures that were described here demonstrate how borescopic IR imaging can be used to obtain quantitative information about early flame kernel growth and how this is related to combustion performance.

\section{References}

1. Sick, V., "High Speed Imaging in Fundamental and Applied Combustion Research," Proceedings of the Combustion Institute 34(2):3509-3530, 2013, doi:10.1016/j. proci.2012.08.012.

2. Sick, V., Drake, M.C., and Fansler, T.D., "High-Speed Imaging for Direct-Injection Gasoline Engine Research and Development," Experiments in Fluids 49(4):937-947, 2010, doi:10.1007/s00348-010-0891-3.

3. Agrawal, G.P., "Fiber-Optic Communication Systems," (New York, John Wiley \& Sons, 1997).

4. Turns, S.R., "An Introduction to Combustion: Concepts and Applications," 3rd Edition (New York, McGrawHill, 2012).

5. Ludwig, C. B., Malkmus, W., Reardon, J., Thomson, J., et al. 1973. Handbook of Infrared Radiation from Combustion Gases. Washington, D.C.: Natioinal Aeronautics and Space Administration.

6. Frank, J., Barlow, R., and Lundquist, C., "Radiation and Nitric Oxide Formation in Turbulent Non-Premixed Jet Flames," Proceedings of the Combustion Institute 28(1):447454, 2000.

7. Brookes, S. and Moss, J., "Measurements of Soot Production and Thermal Radiation from Confined Turbulent Jet Diffusion Flames of Methane," Combustion and Flame 116(1):49-61, 1999.

8. Schiffmann, P., Reuss, D.L., and Sick, V., "Empirical Investigation of Spark-Ignited Flame-Initiation Cycle-toCycle Variability in a Homogeneous Charge Reciprocating Engine," International Journal of Engine Research 146808741 7720558:146808741772055, 2017.

9. Chen, H., Lillo, P.M., and Sick, V., “Three-Dimensional Spray-Flow Interaction in a Spark-Ignition Direct-Injection Engine," International Journal of Engine Research 17(1):129138, 2016.

10. Chen, H., Xu, M., Hung, D.L., and Zhuang, H., "Cycle-ToCycle Variation Analysis of Early Flame Propagation in Engine Cylinder Using Proper Orthogonal Decomposition," Experimental Thermal and Fluid Science 58:48-55, 2014.

11. Hung, D.L., Chen, H., Xu, M., Yang, J. et al., "Experimental Investigation of the Variations of Early Flame Development in a Spark-Ignition Direct-Injection Optical Engine," Journal of Engineering for Gas Turbines and Power 136(10):101503, 2014.

12. Schiffmann, P., "Root Causes of Cycle-To-Cycle Combustion Variations in Spark Ignited Engines.” 2016.
13. Mazacioglu, A., Gross, M., Kern, J., and Sick, V., "Infrared Borescopic Evaluation of High-Energy and Long-Duration Ignition Systems for Lean/Dilute Combustion in HeavyDuty Natural-Gas Engines," SAE Technical Paper 2018-01$\underline{1149}, 2018$.

14. Otsu, N., "A Threshold Selection Method from Gray-Level Histograms," IEEE Transactions on Systems, Man, and Cybernetics 9(1):62-66, 1979.

15. Ayala, F.A. and Heywood, J.B., "Lean SI Engines: The Role of Combustion Variability in Defining Lean Limits," SAE Technical Paper 2007-24-0030, 2007, doi:10.4271/2007-24$\underline{0030}$.

16. Matekunas, F.A., "Modes and Measures of Cyclic Combustion Variability," SAE Technical Paper 830337, 1983, doi:10.4271/830337.

\section{Contact Information}

\author{
Michael C. Gross \\ University of Michigan \\ W. E. Lay Automotive Engineering Laboratory \\ 1231 Beal Avenue \\ Ann Arbor MI 48109-2133 \\ (734) 936-0426 \\ mcgross@umich.edu
}

\section{Acknowledgments}

The information, data, or work presented herein was funded in part by the Office of Energy Efficiency and Renewable Energy (EERE), U.S. Department of Energy, under Award Number DE-EE0007307. The authors also thank Dr. Hao Chen and Angela Wu for their help with software, Dr. David L. Reuss for discussions of combustion and diagnostics, and James Elkins for engine-head modifications.

\section{Definitions and Abbreviations}

$\boldsymbol{A}$ - projected area of the flame in the image

$\boldsymbol{c}_{\boldsymbol{m}, n}$ - pixel intensity at $m^{\text {th }}$ row and $n^{\text {th }}$ column

CAD - crank-angle degree

CCV - cycle-to-cycle variability

CoV(IMEP) - coefficient of variation of IMEP

EGR - exhaust-gas recirculation

$I$ - second moment of projected area of the flame in the image IMEP - indicated mean effective pressure

IR - infrared

$\boldsymbol{m}$ - row index

$\boldsymbol{M}$ - total number of rows in the image

mean $\left(M B_{00.5}\right)$ - the mean value of $\mathrm{MFB}_{00.5}$, evaluated over all cycles of a measurement

$\mathbf{M F B}_{x}$ - the crank angle at which $x \%$ of the fuel mass has been burned, evaluated for one cycle 
$\boldsymbol{n}$ - column index

$N$ - total number of columns in the image

$\boldsymbol{P}_{\text {max }}$ - peak in-cylinder pressure

$\boldsymbol{R}(\boldsymbol{x}, \boldsymbol{y})$ - Pearson correlation coefficient measured between variables $x$ and $y$

$\boldsymbol{r}_{\text {gyr }}$ - radius of gyration

$S$ - total integrated signal in an image $x_{c}$ - location of the centroid on the $x$-axis

$y_{c}$ - location of the centroid on the $y$-axis

$\lambda$ - air/fuel equivalence ratio

$\boldsymbol{\theta}_{x / 5}$ - the crank angle $x / 5$ of the time (in CAD) between spark timing and mean $\left(\mathrm{MFB}_{00.5}\right)$

${ }^{\circ}$ aTDC - degrees after top dead center

All rights reserved. No part of this publication may be reproduced, stored in a retrieval system, or transmitted, in any form or by any means, electronic, mechanical, photocopying, recording, or otherwise, without the prior written permission of the copyright holder.

Positions and opinions advanced in this paper are those of the author(s) and not necessarily those of SAE International. The author is solely responsible for the content of the paper.

ISSN 0148-7191 